\section{REVISTA FAECO SAPIENS \\ ISSN L 2644-3821}

Acceso Abierto. Disponible en:

https://revistas.up.ac.pa/index.php/faeco_sapiens

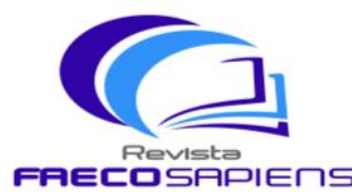

Indexada

\title{
EFECTOS DE LA NIIF PARA PYMES EN LAS EMPRESAS DE CONSTRUCCIÓN DE EDIFICIO EN PANAMA
}

Effects of the IFRS for SMES on building construction companies in Panama

\author{
Alejandro Antonio Rodríguez Santos \\ Facultad de Administración de Empresas y Contabilidad, Universidad de Panamá \\ Email: arodrigu15@hotmail.com iD https://orcid.org/0000-0001-6832-5555
}

\begin{abstract}
RESUMEN
Este artículo evalúa los efectos de la implementación de la Norma Internacional de Información Financiera para Pequeñas y Medianas Empresas en las empresas de construcción de edificio registradas en el distrito de la ciudad de Panamá. Para el desarrollo de la investigación se consideró una población que representa la muestra de 79 empresas. La metodología utilizada, consistió en dos encuestas que se realizó con el diseño de pregunta con escala Likert para hacer la recogida de la información más eficiente, aplicadas a los dueños o gerentes y colaboradores del área de contabilidad de una prueba pilotos de 12 personas que forman parte de la población de 79 empresas. Los datos fueron analizados con la ayuda del programa estadístico SPSS. Los resultados obtenidos, evidenciaron una contabilidad confiable, mejoras en la calidad de la información para la preparación de los estados financieros para la toma de decisiones económica y financiera. Como conclusión, la implementación de la Norma Internacional de Información Financiera para Pequeñas y Medianas Empresas conlleva transparencia en su actividad financiera, comparabilidad y confiabilidad de sus informes financieros, competitividad en los mercados y cumplimiento de regulaciones legales nacionales e internacionales
\end{abstract}

Palabras clave: Norma internacional de información financiera para pequeñas y medianas empresas, contabilidad confiable, mejoras en la calidad de la información, estados financieros transparente, cumplimiento de regulaciones.

\begin{abstract}
This article assesses the effects of the implementation of the International Financial Reporting Standard for Small and Medium Enterprises on registered building construction companies in the Panama City district. For the development of the research, a population representing the sample of 79 companies was considered. The methodology used consisted of two surveys that were carried out with the Likert scale question design to make the collection of information more efficient, applied to the owners or managers and collaborators of the accounting area of a pilot test of 12 people who form part of the population of 79 companies. The data was analyzed with the help of the SPSS statistical program. The obtained results evidenced a reliable accounting, improvements in the quality of the information for the preparation of the financial statements for economic and financial decision making. In conclusion, the implementation of the International Financial Reporting Standard for Small and Medium-Sized Companies implies transparency in their financial activity, comparability and reliability of their financial reports, competitiveness in the markets and compliance with national and international legal regulations.
\end{abstract}

Keywords: International financial reporting standard for small and medium-sized businesses, reliable accounting, improvements in the quality of information, transparent financial statements, regulatory compliance. 

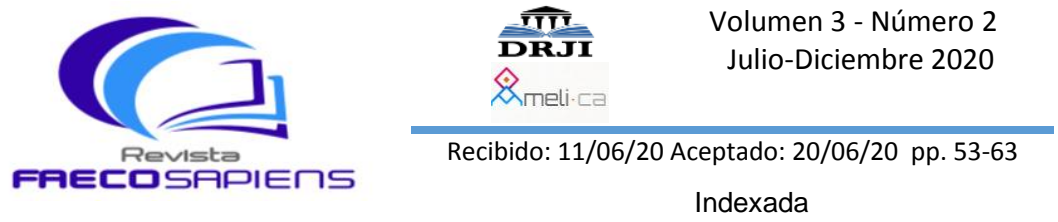

INTRODUCCIÓN

Gracias a la globalización las empresas se han visto en la necesidad de implementar las NIIF para contar con un mismo lenguaje financiero que contribuye a nuevas oportunidad y ampliación de mercados. Igualmente, las pequeñas y medianas empresas no se han hecho esperar al deseo de implementar las NIIF para pymes aunque mantienen cierto recelo al respecto; por otra parte, las pyme son los pilares de la economía incluso en los países desarrollados. El siguiente artículo pretende mostrar los efectos de la implementación de las NIIF Pyme en algunas de las pequeñas y medianas empresas del sector de la construcción de edificio en Panamá que se tomaron como muestra.

En Panamá aún es reciente el tema de las NIIF Pyme por lo cual aún no existe mucha información local sobre el tema, en este artículo se toma como referencia varios documentos de otros países donde existe más datos al respecto y que sirvieron como base documental para el desarrollo de este artículo.

\section{El Impacto de la Globalización}

La globalización, es un proceso de cambio en las relaciones internacionales, el solo hecho de validar los tratados de libre comercios que se dan entre los países son escenarios de estas características, sin embargo, frente a estas situaciones surgen inconvenientes con el idioma al no contar con un lenguaje internacional para la presentación de información financiera, lo que se hace necesario en un mercado globalizado.

Según Arias Bello (2011), La apertura de los mercados en el proceso de globalización ha impuesto importantes exigencias para aquellos que buscan expandir sus negocios. En procura de generar un lenguaje común en el contexto de ese mercado global, las normas internacionales de información financiera se han convertido en prioridad de regulación contable en muchos escenarios económicos (p.1)

\section{Las NIIF Pymes}

El Comité o Junta de Normas Internacional de Contabilidad conocido por su siglas en inglés IASB (International Accounting Standard Board), organismo independiente que nace del sector privado, responsable de la emisión de las Normas Internacionales de Información Financieras NIIF, lo que ha buscado es crear un camino para que todas las empresas en el mundo adopten las Normas Internacional de Información Financiera (NIIF). 

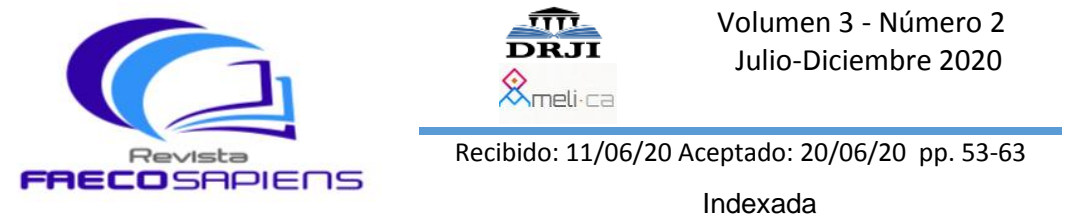

En julio de 2009 la Juna de Normas Internacional de Contabilidad (IASB), al ver la necesidad que presentaban las PYMES de competir en los mercados internacionales emite la Normas Internacional de Información Financiera para Pequeñas y Medianas Empresas (NIIF para PYMES) en base a las NIIF plena, con el propósito de que al ser aplicadas en estas empresas para que logren una información financiera de alta calidad y transparente, comparabilidad de los informes financieros con otros países y la oportunidad de participar y competir en los mercados internacionales.

\section{Implementación NIIF Pymes y Pymes en Panamá}

Al implementarse la NIIF para PYMES, la Ley 72 de 9 de noviembre de 2009, reforma la Ley 8 del 29 de marzo de 2000 y la Ley 33 del 2000, que aplica dependiendo el tipo de PYMES (Ley 33, 2000).

Luego el diario, El Panamá América Periódico local, del martes 26 agosto 2019. Informó que: Las Micro y Pequeñas Empresas en Panamá, es el segmento que representa el $99 \%$ de las empresas formales en Latinoamérica y generan el $61 \%$ de empleo formal, sin embargo, no se afirma sobre el cumplimiento de la aplicación de la NIIF para PYMES (Panamá América, 2019). Es importante resaltar que las PYMES son importantes no sólo en Panamá sino en todos los países, ya gracias a que estas pequeñas y medianas empresas son las generadoras de empleos y de apoyo al crecimiento económico de los países

Existen varios motivos para crear una PYMES, entre los que se encuentran el emprendimiento de muchos para generación de ingresos, otros motivados por el aspecto económico del país y a nivel mundial (Pascual, Héctor, \& Leandra, 2013).

De acuerdo a lo anterior algunos autores creen que las microempresas son quienes ayudan a reducir la pobreza, al generar empleos y al apoyar el crecimiento social, sabiendo que para esto es necesario que estas PYMES reciban asesorías y capacitación para poder acceder a mercados adicionalmente cuenten con apoyo financiero o acceso a estos (Jocelyne Ramírez \& Moctezuma, 2014); es por esto que es importante la participación para la implementación de una asesoría para la adopción de la NIIF para PYMES para que estas empresas tengan la oportunidad y puedan incursionar en nuevos mercados y acceder a créditos para mejorar su capital financiero y capital de trabajo.

Por lo tanto, esta investigación tiene como propósito evaluar los efectos de la implementación de la NIIF para PYMES, en las medianas empresas del sector de la construcción de edificio en Panamá. Permitiendo así evaluar, conocer, y 
REVISTA FAECO SAPIENS

ISSN L 2644-3821

Acceso Abierto. Disponible en:

https://revistas.up.ac.pa/index.php/faeco_sapiens

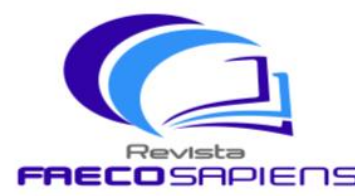

ค月EடロSคคIEกร

establecer los efectos que tendrán las empresas con la aplicación de la Norma Contable.

Bajo este enfoque, el proyecto de investigación está orientado a dar respuesta a la interrogante que se plantea en la formulación del problema, considerando la determinación de cómo se está manejando el flujo de la información financiera actualmente y cuáles son los cambios y estrategias que están aceptando los directivos para hacerle frente a esta situación que requiere de un trabajo planeado y organizado que tenga como fin cumplir con las nuevas exigencias de la nueva norma.

A continuación, estaremos presentando informaciones que permitan a las empresas conocer los procesos de la aplicación de las NIIF para PYMES con el fin de incorporar la voluntad de los participantes, para hacer de esta experiencia un proceso eficiente y enriquecedor para todos.

Aunque es bien conocido que las medianas empresas cuentan con poco empleados, ingresos moderados y siempre buscan un crecimiento económico constante y están sometidas a la AMPYMES desde mayo del 2000 (Ley 8, 2000); al entrevistar varias PYMES del sector de la construcción de edificios en Panamá se busca identificar los efectos buenos o no al implementar las NIIF para PYMES y sobre todo lo que puedan describir el proceso de ésta implementación, el apoyo o asesoría sobre este tema para contar con más argumentos sobre el nivel de conocimiento con que cuentan en Panamá las pequeñas y medianas empresas sobre la aplicación de las NIIF para PYMES.

Este proyecto de investigación es importante porque expone de forma descriptiva la evaluación de la implementación de la NIIF para PYMES, en las medianas empresas del sector de la construcción de edificio en Panamá, a fin de conocer los efectos que trae consigo y que se ve reflejado en la información financieras para preparar los estados financieros, logrando en las empresas transparencia, información de calidad, objetividad y oportuna; lo que permite a sus dirigentes tomar decisiones de carácter estructural, financiero o de proceso en el momento justo para que mejore los resultados de la empresa y sobre todo ahora donde el sector de la construcción en Panamá ha bajado su participación en un 10,5\% del PIB tan solo el año pasado lo que hace necesario contar con este tipo de estados financieros que ayuden a la autoevaluación de la empresa y ayude a la toma de decisiones que beneficien a la empresa.

Por lo tanto, esta investigación servirá de apoyo a las Medianas Empresas del sector de la construcción de edificio en la ciudad de Panamá, estableciendo conocimientos necesarios para que impulse al fortalecimiento y crecimiento de 

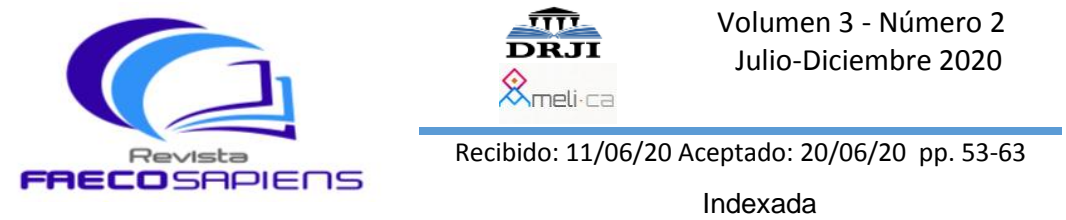

estas empresas, así como también dejándolo como medio de consulta para que otras pequeñas y medianas empresas que no siendo del sector de la construcción lo puedan aprovechar y conocer cómo se puede utilizar la implementación de las NIIF para PYMES.

Por esta razón es importante realizar una investigación aplicada de campo donde la técnica para recabar los datos se realice a través de encuestas a los empresarios y contadores responsables de las medianas empresas constructoras en Panamá, ya que, la finalidad es identificar cuáles son efectos que les ha causado la implementación de la NIIF para PYMES, adicionalmente se podrá determinar; la cantidad de empresas de este sector que han adoptado, y las que las están adoptándolas por primera vez la NIIF para PYMES, el conocimiento que se tienen sobre estas normas y las dificultades que han tenido que enfrentar en este proceso, más un concepto personal sobre si sienten que les ha servido o no la implementación de la NIIF para PYMES

La metodología será una investigación no experimental transversal descriptiva correlación y explicativa, en donde las variables no se manipulan tal como lo señala (Hernández, Fernández, \& Baptista, 2014), de que las investigaciones no experimentales no se manipulan las variables. Ya que, lo que se pretende es analizar la relación que existen entre la variable independiente y la dependiente con fin describir su comportamiento y explicar su relación sin manipular las variables, a fin de conocer los efectos contables y los resultados encontrados sobre la aplicación de la NIIF para PYMES.

La implementación de la NIIF para PYMES trae consigo reto y oportunidades para las empresas interesadas en evolucionar y salir de su área de confort y ser competitiva internacionalmente en los mercados de capitales.

Al implementar la NIIF para PYMES en su sistema contable permitirá que su información financiera sea interpretada internacionalmente contando con lenguaje contable común a nivel mundial.

En relación a este tema, Criado, Rangel \& Solano (2014), En su el artículo científico señalan que; La convergencia a la NIIF para PYMES, es un paso para internacionalizar la economía regional, ya que dichos estándares son aplicados en una gran cantidad de países y su objetivo es generar información financiera de alta calidad y comparable a través del tiempo del mismo ente y con las demás empresas que conforman los diferentes sectores económicos.

Para Criado, et at (2014) en su artículo científico detalla cuatro fases: capacitación, diagnósticos y utilización de recursos, elaboración de políticas contables y elaboración del estado de situación financiera de apertura, se aplicó 

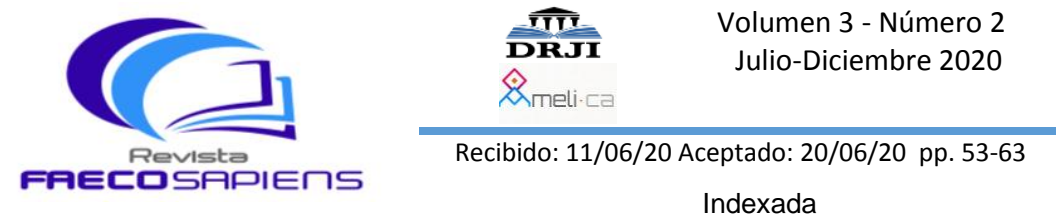

una encuesta a funcionarios del área contable o financiera den sector industria de la ciudad donde arrojó sus resultados que revelaron la falta de compromiso desde la administración de las organizaciones y han generado gran impacto no solo en las áreas contables y financieras, sino en todas las áreas de las diferentes organizaciones.

Por otro lado Salazar Baquero (2013), en su estudio titulado "Efectos de Implementación de NIIF para PYMES para la en una mediana empresa ubicada en la ciudad de Bogotá Pontificia Universidad Javeriana, Bogotá. Su metodología fue un estudio de caso intensivo Especialista en gerencia y administración tributaria, Universidad Externado de Colombia en su artículo expone un estudio de casos en el cual se implementó la NIIF para PYMES en una mediana empresa colombiana los resultados evidencian mejoras en la calidad de información contable al tiempo que señala las dificultades que pueden experimentar una entidad con la implementación de la NIIF para PYMES llegando a la conclusión determinar distintos tipos de efectos financieros, sus elecciones depolit6iocas contable y la precisión en sus estimaciones contables (p.p. 2.16).

Para Lozada Rivera (2015) en su tesis doctoral denominada" Comparación del valor de pertenencia de las normas contables en Estados Unidos versus las normas internacionales financiera el caso de las American Depositary Receipts" Universidad de León España, donde detalla En este estudio se ha examinado el valor de pertinencia y la utilidad que les brinda a los inversionistas la divulgación de la cifra de resultado cuando las empresas comunican su información financiera utilizando las Normas Internacionales de Información Financiera (NIIF) y las normas contables estadounidenses, conocidas como U.S. GAAP. Se ha investigado en determinadas empresas que han utilizado ambas normas, NIIF o U.S. GAAP, y los resultados de la comparación influye en la determinación de los precio de las acciones. La finalidad de esta comparación era determinar que normativa contable provee mayor valor de pertinencia.

Encalada, Castillo \& Ruiz (2019), en su artículo científico concluyen considerando que; La estructura interna y externa de manera general y particular, los elementos comunes de la implementación de la NIIF para las PYMES es posible que la NIIF para las PYMES obliga a la gerencia a revelar una mejor calidad de la información económico-financiera. Además, proporciona de manera amplia y razonable, por una parte, información exclusiva para inversionistas y proveedores, por otra parte, revela información para asuntos de política fiscal, es decir, ayuda a separar información entre lo contable y tributario. La implementación de la NIIF representa un apoyo a los propósitos internos de la PYMES por alta calidad de la información. Finalmente, de la efectiva información proporcionada en los nuevos balances, la entidad conoce las actuales políticas contables, estimaciones y errores (p.17). 

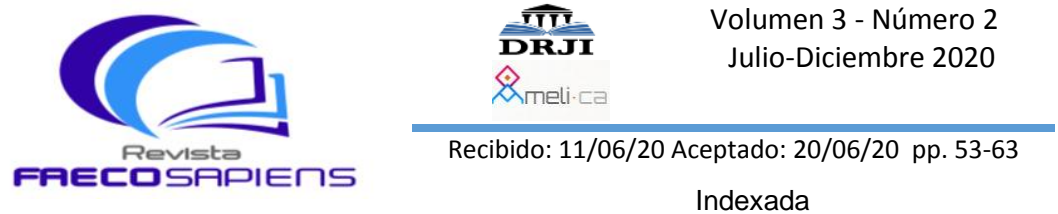

Según Mantilla Blanco (2017). En su libro Entendiendo el estándar NIIF para PYMES señala; son estándares internacionales para las pequeñas y medianas entidades, emitidas por la Junta de Normas Internacionales de Contabilidad (IASB) en 2009, incorporan principios basados en las NIIF plenas pero simplificados para las PYMES.

Cabe agregar que este mismo autor, usando el método de pregunta y respuesta, gráficas y tablas, propone un completo recorrido por la última versión del estándar y sus elementos: definiciones básicas, estados financieros, instrumentos financieros, activos, pasivos, patrimonio, provisiones y la transición al estándar, consideración a la estructura interna y externa de manera general y particular, los elementos comunes de la implementación de la NIIF para las PYMES es posible revelar que la NIIF para las PYMES obliga a la gerencia una mejor calidad de la información económico-financiera. Además, proporciona de manera amplia y razonable, por una parte, información exclusiva para inversionistas y proveedores, por otra parte, revela información para asuntos de política fiscal, es decir, ayuda a separar información entre lo contable y tributario. La implementación de la NIIF representa un apoyo a los propósitos internos de la PYMES por alta calidad de la información. (p.1).

Según (Byard, Li, \& Yu, 2010), analizaron que los efectos de la adopción obligatoria de las Normas Internacional de Información Financiera (NIIF) por las empresas de la Unión Europea, presentaron resultados favorables e importantes en cuanto a la transparencia de los informes financieros

(Latridis \& Rouvolis, 2009), señalan que los efectos de la adopción de las NIIF para las empresas griegas que cotizan en bolsa de valores el primer año los resultados no fueron favorables debido a los costos de transición de las NIIF, sin embargo, para periodo posteriores los resultados mejoraron significativamente. Por lo tanto, la adopción de las NIIF conduce a medidas contables relevantes para el valor razonable.

Según (Sánchez Holguín, 2017) comenta que; Es posible que la implementación de la NIC 11: Contratos de Construcción y de la Sección 23 de las NIIF para PYMES haya arrojado resultados o impactos negativos, pero a medida que las empresas logren ver la importancia que tienen estas normas en el reconocimiento de los ingresos y los costos, se va a notar como su información va a ser más verídica y van a poder tomar mejores decisiones.(p.21)

En el mismo orden de idea (Sánchez Holguín, 2017) concluye señalando que; A pesar de todos los cambios y posibles obstáculos que se puedan llegar a presentar en la implementación de las normas internacionales, estos nuevos 

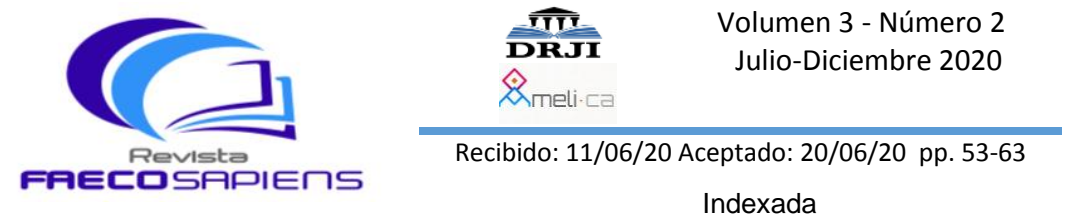

estándares tienen como propósito darle un mejor direccionamiento a las grandes, medianas y pequeñas empresas, ya que el objetivo de estas normas es prescribir el tratamiento contable de los ingresos de actividades ordinarias y los costos relacionados con los contratos de construcción.

En estos antecedentes se destaca la relación con el proyecto de investigación en cuanto la importancia que tiene la implementación de las NIIF en las PYMES en las medianas empresas, para alcanzar los resultados esperados en el ámbito contable y financiero.

Para concluir, estos antecedentes citados están ligados directamente con el proyecto de investigación, por lo tanto, servirán como aportes significativos, para facilitar el análisis de los efectos de la implementación de la NIIF para PYMES en las medianas empresas del sector de la construcción ubicada en Panamá.

\section{MATERIALES Y MÉTODOS}

Este artículo investigativo se realiza con un enfoque cuantitativo y cualitativo de tipo descriptivo mediante una revisión documental de varios libros, artículos científicos y los resultados de algunas encuestas realizada a Empresarios del sector de la construcción y contadores que son en realidad los que viven los efectos de esta implementación de forma directa.

Esta investigación se realiza en 3 etapas que son: 1. Recolección de información, para luego seleccionar los que aporten a la investigación y que no sobrepasen los 10 años con el fin de contar con información actualizada. 2. Realización de entrevistas que proporcionara información importante sobre los efectos de la implementación de las NIIF pyme en sus respectivas empresas. 3. Análisis de la información recopilada después de pasar por las etapas 1 y 2 .

\section{RESULTADOS Y DISCUSIÓN}

Después de analizar la información recopilada y los resultados de las encuestas se ha determinado que los efectos son los siguientes:

1. Costos de implementación, estos son los gastos que los empresarios asumen antes y durante la implementación de las NIIF pymes, como son la capacitación sobre las normas ya que deben contar con personal idóneo para realizar el cambio a las NIIF. Aunque se pueda ver como un efecto negativo estos costos son importantes para la empresa ya que permite el desarrollo de la implementación de las NIIF con el fin de evitar cometer errores que más adelante 

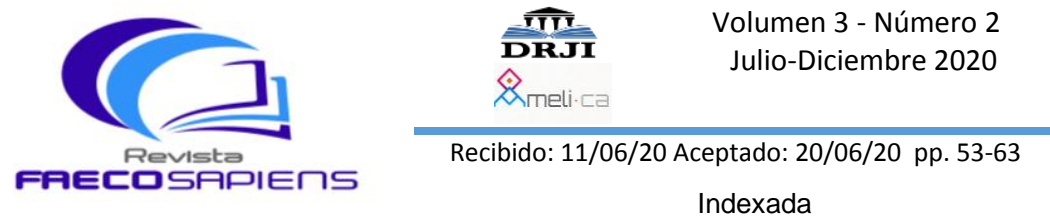

conllevará a un gasto realmente elevado por fallas o mala implementación que se puede deber a falencias o desconocimiento de la norma completamente.

2. Los efectos financieros que deja la implementación y que afecta las cuentas de: Impuestos Diferidos, los ajustes a las cuentas por cobrar a largo plazo, la cuenta de activos fijos, el deterioro del valor de las inversiones y en propiedad planta y equipo.

3. En los efectos operativos se puede decir que están de acuerdo a lo investigado en la elaboración de los estados financieros donde se puede resaltar que una de las complicaciones sería el determinar la tasa de interés para créditos a largo plazo, ya que se cuenta con varias tasas que se pueden utilizar. Por otro lado, el determinar la vida útil de los activos; estos estados financieros hacen que se analice con mayor detenimiento ya que las NIIF Pymes comprenden un análisis con cálculos retroactivos que exigiría mayor conocimiento de las normas internacionales de contabilidad.

4. De acuerdo a la parte tributaria se encuentran inicialmente algunas falencias de interpretación de las normas y algunos aspectos que no cuentan con la interpretación como en los inventarios los descuentos financieros que no son regulados y no deja claro si es aplicable a los efectos fiscales. Igualmente se incurre en más esfuerzo laboral debido a que son más informes que se debe preparar y que es originario a los saldos de contabilidad.

5. En la parte administrativa se puede evidenciar que aunque aún es algo novedoso se tiene ciertas dudas sobre si realmente es algo positivo para la empresa lo que hace que no todas las pyme de este sector cuente con la implementación de estas normas, según lo expresado por los empresarios en las entrevistas, algunos de éstos están a la espera de los resultados y los cambios que las otras que si las han implementados inicien a mostrar para poder tomar una decisión

Para concluir se puede evidenciar que son varios los efectos que acarrea la implementación de las NIIF pymes pero que igualmente se puede dejar claro que es una nueva forma de entrar a ser más competitivas estas pequeñas y medianas empresas que como todas quieren llegar a otros mercados y más el sector de la construcción que es uno de los que más aporta al PIB en muchos países del mundo. 
REVISTA FAECO SAPIENS

ISSN L 2644-3821

Acceso Abierto. Disponible en:

https://revistas.up.ac.pa/index.php/faeco_sapiens

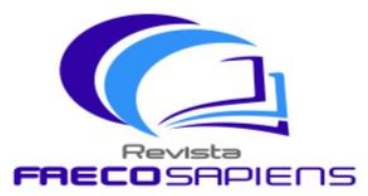

DRJI

Volumen 3 - Número 2 Julio-Diciembre 2020

Recibido: 11/06/20 Aceptado: 20/06/20 pp. 53-63

Indexada

\section{BIBLIOGRAFÍA}

Arias-Bello, M. (julio -diciembre de 2011). Reflexiones sobre la Contabilidad de activos financieros en el conetexto del modelo IASB y el contexto actual colombiano. Cuad.Cont./Bogotá, Colombia, 12(31):469-490/julio -diciembre 2011. Obtenido de file:///C:/Users/Alejandro/Downloads/3099-Texto\%20del\%20art\%C3\%ADculo-10943-1-1020120801.pdf.

Byard, D., Li, Y., \& Yu, Y. (22 de octubre de 2010). El efectod de la adopción obligatoria de las NIIF en el entorno de información de loa anlista financieros. (J. o. Rsearch, Editor) Obtenido de Jounal of Accounting Rsearch: https://dol.org./10.1111/j.1475679X.2010.00390

Criado Alzate, Y., Rangel Castilla , A., \& Solano Becerra, E. (2014). Estudio de las pequeñas empresas de Cúcuta sobre la convergencia a las normas internacionales de información financiera para PYMES. Repuestas, 6-14. Obtenido de https://revistasufpseduco.biteca.online/index.php/respuestas

Encalada E, V. R., Castillo C, A. L., \& Ruiz Q, S. C. (2019). Efectos de la Implemenacion de la Normas Internacional de Información Financiera en una peqpeña Empresa registrada en la ciudad de Guayaquil, Ecuador. Obtenido de Pontificia Universidad Javeriana: https://do.org./10.11144/javeriana.cc20-50.eini

Hernández, S. R., Fernández, C., \& Baptista, L. P. (2014). Metodología de la Investigación (6o Edición ed.). Mexico: McGraw-Hill / Interamericana Editores, S.A, Torre A. Obtenido de www. Itercambiosviertuales.org.

Jocelyne Ramírez, R., \& Moctezuma, M. B. (2014). Income Inequality and Its Determinants in Microenterprises in Baja California, México. En J. o. Management. México.

La Prensa. (6 de agosto de 2019). Capacitación Claves en las Pymes. Obtenido de prensa.com: https://www.prensa.com/economia/Capacitacion-clavespymes_0_5367213305.html

Latridis, G., \& Rouvolis, S. (4 de diciembre de 2009). Los efectos posteriores a la adopción de la implementación de las Normas Internacionales de Información Financiera en Grecia. doi:DOI: 10.1016 / j.intaccaudtax.2009.12.004

Ley 33. (25 de julio de 2000). Calificaión Pyme. Ley que define el concepto de micro, pequeña y mediana empresa según su ingreso anual. Ciudad de Panamá.

Lozada Rivera, A. R. (6 de marzo de 2015). Comparación del valor de pertinencia de las normas contables en Estados Unidos versus las normas internadcional de información financiera el caso de las American Depositary Receipts. Comparación del valor de pertinencia de las normas contables en Estados Unidos versus las normas internadcional de información financiera el caso de las American Depositary Receipts. España, León España. Obtenido de https://buleria.unileon.es/bitstream/handle/10612/4555/tesis_f2ac65\%20(1).PDF?seq uence $=1$

Mantilla Blanco, S. A. (2017). Entendiendo el estándar NIIF para Pymes. Ecoe Ediciones. Obtenido de http://www ecoediciones.com/libro de contabilidad y finanza/ebook

Panamá América. (26 de agosto de 2019). Micro y pequeña empresa es el sector con mayor generación de empleos. Obtenido de https://www.panamaamerica.com.pa/economia/micro-y-pequena-empresa-es-elsector-con-mayor-generacion-de-empleos-587634

Pascual, B., Héctor, G., \& Leandra, G. R. (6 de agosto de 2013). Determinants of 
REVISTA FAECO SAPIENS

ISSN L 2644-3821

Acceso Abierto. Disponible en:

https://revistas.up.ac.pa/index.php/faeco_sapiens

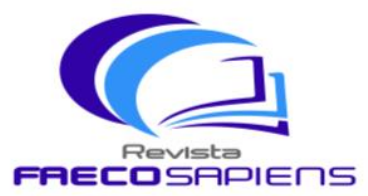

पाT Volumen 3 - Número 2 DRJI Julio-Diciembre 2020

多meli.ca

Recibido: 11/06/20 Aceptado: 20/06/20 pp. 53-63

Indexada

Performance in microenterprises: Preliminary Evidence from Argentina. Obtenido de Journal of Bussines: https://onlinelibrary.wiley.com/doi/abs/10.1111/jsbm.12045

Salazar - Barquero, É. E. (julio -diciembre de 2013). Efectos de la implementación de la NIIF para las PYMES en una mediana empresa ubicada en la ciudad de Bogotá. (C. 1. Cuaderno de Conatabilidad - Bogotá, Ed.) Cuaderno de Conatabilidad - Bogotá, Colombia, 14 (35) , 395-414. Obtenido de http://revista javeriana edu.co./;index.php/cuacon/artocñe/view/7097

Sánchez Holguín, L. S. (2017). IMPLEMENTACIÓN DE LAS NIIF EN LAS EMPRESAS DEDICADAS A LOS CONTRATOS DE CONSTRUCCIÓN PARTIENDO DE LA NIC 11 Y LA SECCIÓN 23 DE LAS NIIF PARA PYMES. IMPLEMENTACIÓN DE LAS NIIF EN LAS EMPRESAS DEDICADAS A LOS CONTRATOS DE CONSTRUCCIÓN PARTIENDO DE LA NIC 11 Y LA SECCIÓN 23 DE LAS NIIF PARA PYMES., 25. Bogotá, Colombia, Bogotá, Colombia: Universidad Militar Nueva Granada Facultad De Ciencias Económicas Contaduría Pública. Obtenido de https://repository.unimilitar.edu.co/bitstream/handle/10654/17232/SanchezHolguinLa dyStefany2017.pdf; 